\title{
Long-term effects of lifestyle on multiple risk factors in male workers
}

\author{
Hanayo Koetaka $\cdot$ Yuko Ohno $\cdot$ Kanehisa Morimoto
}

Received: 16 April 2008/Accepted: 19 December 2008/Published online: 7 March 2009

(C) The Japanese Society for Hygiene 2009

\begin{abstract}
Objectives To examine the long-term effects of lifestyle on the recovery from risk factors of cardiovascular disease and to discuss the difference in the effects of lifestyle modification in subjects with a single risk factor and those with multiple risk factors.

Methods We used checkup data compiled for 6477 male workers, aged 20-59 years in 1995, with risk factors of cardiovascular disease. The relation between the recovery from risk factors 9 years later and baseline lifestyles was examined by logistic regression according to the initial number of risk factors.

Results Nine years following the baseline measurements, 1907 subjects had recovered from at least one risk factor. When there was initially a single risk factor, a good overall lifestyle was effective in the recovery [odds ratio (OR) 1.27; 95\% confidence interval (CI) 1.03, 1.57], with maintaining good dietary habits (OR 1.24; 95\% CI 1.07, 1.45 ) and moderate stress levels (OR 1.19; 95\% CI: 1.03 , 1.38 ) both found to be especially effective in the recovery. When there were multiple risk factors, although the effect of a good overall lifestyle on the recovery was less than that when there was only a single risk factor, non-smoking
\end{abstract}

H. Koetaka - Y. Ohno

Department of Mathematical Health Science, Osaka University Graduate School of Medicine, Suita, Osaka, Japan

\section{K. Morimoto $(\square)$}

Department of Social and Environmental Medicine,

Osaka University Graduate School of Medicine,

2-2 Yamadaoka, Suita, Osaka 565-0871, Japan

e-mail: morimoto@envi.med.osaka-u.ac.jp
(OR 1.27; 95\% CI 1.07, 1.51) and limiting working hours (OR 1.25; 95\% CI 1.05, 1.49) were found to be effective. Conclusions Our results provide evidence that good lifestyles are effective in the recovery from multiple risk factors. Effects of lifestyle on recovery from multiple risk factors are different from effects on the recovery from a single risk factor, with the difference depending on the initial number of risk factors.

Keywords Health practice - Lifestyle . Long-term effects · Longitudinal studies . Metabolic syndrome . Workers

\section{Introduction}

Metabolic syndrome is defined as a group of risk factors, such as obesity, hypertension, dyslipidemia, hyperglycemia, and other metabolic abnormalities, that can lead to the development of serious diseases [1,2]. The etiology of metabolic syndrome is complex, as it is determined by the interplay of both genetic and environmental factors, such as lifestyle [3]. The long-term effects of lifestyle on the prevention of a single risk factor are known [4-6], and it is believed that a good lifestyle can prevent metabolic syndrome. However, how lifestyles affect metabolic syndrome would differ from how they affect a single risk factor because metabolic syndrome is defined as a group of multiple risk factors. Morimoto et al. examined the relationship of life style to sister chromatid exchange [7], natural killer cell activities [8-10], mutagen levels excreted in the urine [11], and immunoglobulin E [12]. These researchers provided evidence that a good lifestyle is influential in the prognosis of lifestyle-related diseases, such as cancer and allergic disorders. Within the context of 
preventing metabolic syndrome, it is important to examine the long-term effects of lifestyle on a group of multiple risk factors and to clarify the effects of a good lifestyle. Many studies of metabolic syndrome have been reported recently on populations worldwide. In terms of the influences of lifestyle on metabolic syndrome, however, few studies have followed a large cohort over a long-term period [13, 14], although several cross-sectional studies have examined the relationship between lifestyle and metabolic syndrome [15-17], and another study has investigated the effects of lifestyle intervention on metabolic syndrome improvement in patients with impaired plasma glucose tolerance [18]. In addition, almost all of these earlier studies primarily focused on individual health practices, such as smoking. Considering the complexity of human lifestyles, the effect of overall lifestyle on a group of multiple risk factors should be evaluated.

We have examined the long-term influences of overall lifestyle and individual health practices on the recovery from a group of multiple risk factors and discuss the different effects of these influences on a single risk factor and multiple risk factors, respectively.

\section{Materials and methods}

\section{Subjects}

In Japan, employees generally undergo regular annual checkups at their workplace or at workplace-designated clinics. This study used checkup data collected and managed by a Japanese foundation that performs medical checkups under contract from various employers. The checkups consisted of a physical examination, blood pressure measurements, laboratory analyses of fasting blood samples, and a questionnaire.

In 1995, 106,334 male workers aged 20-59 years underwent checkups. Of these 106,334 subjects, 27,601 also underwent a checkup in 2004. We excluded subjects with missing data on body mass index (BMI), blood pressure, triglycerides or high-density lipoprotein-cholesterol, fasting blood glucose, and eight lifestyles and those who were in treatment for hypertension, dyslipidemia, or hyperglycemia in 1995 . The data from 13,394 subjects were therefore analyzed.

\section{Risk factors}

The risk factors studied were obesity, hypertension, dyslipidemia, and hyperglycemia. In choosing each risk factor, we followed the criteria of the Japan Society for the Study of Obesity, Japanese Society of Hypertension, Japan
Table 1 Criteria for each risk factor

\begin{tabular}{ll}
\hline Risk factor & Criteria \\
\hline Obesity & Body mass index $\geq 25 \mathrm{~kg} / \mathrm{m}^{2 \mathrm{a}}$ \\
Hypertension & $\begin{array}{l}\text { Blood pressure } \geq 140 / 90 \mathrm{mmHg} \\
\text { or a self-reported history of treatment } \\
\text { for hypertension }\end{array}$ \\
& $\begin{array}{l}\text { Triglycerides } \geq 150 \mathrm{mg} / \mathrm{dl}^{\mathrm{c}} \\
\text { or high-density lipoprotein cholesterol } \\
\text { level }<40 \mathrm{mg} / \mathrm{dl},\end{array}$ \\
Dyslipidemia & or a self-reported history of treatment \\
& for dyslipidemia \\
& Fasting blood glucose $\geq 126 \mathrm{mg} / \mathrm{dl}^{\mathrm{d}}$ \\
& or a self-reported history of treatment \\
& for diabetes
\end{tabular}

a The Japan Society for the Study of Obesity

b Japanese Society of Hypertension

c Japan Atherosclerosis Society

d Japan Diabetes Society

Atherosclerosis Society, and Japan Diabetes Society (Table 1). The number of risk factors each subject had in 1995 and 2004, respectively, and the differences in the number of risk factors between these years were calculated. Subjects were classified into five categories according to the number of risk factors they had in 1995: zero risk factors (no risk factor), one risk factor (a single risk factor), and two, three, or four risk factors (multiple risk factors).

To examine the influences of lifestyle in terms of the initial number of risk factors, relations between the decrement in the number of risk factors after 9 years and initial lifestyle were analyzed in the categories of one (single risk factor) to four (multiple risk factors) risk factors. The effects of lifestyle were also analyzed in groups of categories of two to four risk factors $(\geq 2)$ and three to four risk factors $(\geq 3)$ to examine their influences on a group of multiple risk factors and severe multiple risk factors.

Eight health practices and health practice index

Lifestyle factors were assessed from a self-administered questionnaire that asked about eight health practices that had been used by Morimoto et al. in their earlier studies on lifestyle [7-9, 11, 19]. Subjects answered the eight questions shown in Table 2 by either "true" or "false". For each factor, a score of 1 was given for true and 0 for false. In addition, the health practice index (HPI) was calculated by summing scores for the eight health practices to give a total score ranging from 0 to 8 points. Subjects were classified into three categories by the HPI: good health practices (6-8 points), moderate health practices (4-5 points), and poor health practices ( $0-3$ points). 
Table 2 Good health practices in eight health practices

Not smoking
Not drinking more than $46 \mathrm{~g}$ ethanol per day every day
Eating breakfast every morning
Sleeping $7-8 \mathrm{~h}$ per night
Working $9 \mathrm{~h}$ or less per day
Exercising at least twice a week
Eating a nutritionally balanced diet
Keeping a moderate level of mental stress

Each factor was scored 1 for true and 0 for false. The health practice index (HPI) was calculated by summing the scores for the eight health practices. HPI categories were good health practice (6-8 points), moderate health practice ( $4-5$ points), and poor health practice $(0-3$ points)

\section{Statistical analysis}

Two logistic regression models were used in six groups of risk factors $(1,2,3,4, \geq 2, \geq 3$, respectively) to evaluate relations between the recovery from risk factors and lifestyles. We defined recovery from risk factors as a decrease of at least one risk factor in the years between 1995 and 2004 and non-recovery as an increase/no change in the number of risk factors. Using Model 1, we examined the influence of overall lifestyle on the recovery from risk factors. The HPI, age, and the presence of risk factors (obesity, hypertension, dyslipidemia, and hyperglycemia) in 1995 were included. Age was treated as a categorical variable classified in 10-year cohorts. Risk factors were treated as categorical variables (normal or abnormal for each risk factor according to the criteria in Table 1). The HPI was treated with three categorical variables. Odds ratios (ORs) and 95\% confidence intervals (CIs) were computed for the good health practice group and the moderate health practice group of the HPI, and the poor health practice group was used as the reference group. A linear trend across categories was tested with the HPI score treated as a continuous variable. Using Model 2, we examined the influences of the eight health practices on the recovery from risk factors. A stepwise logistic regression analysis with $P<0.20$ for inclusion as a determining factor was performed. The eight health practices (good health practice or poor health practice), age, and the presence of risk factors in 1995 were included. Variables other than health practices were treated as in Model 1. The ORs and CIs were computed for the good health practice group of each health practice, and the poor health practice group was used as the reference group. All analyses were performed using SAS statistical software, ver. 8 (SAS Institute, Cary, NC).

This study was approved by the ethical committee of the Course of Health Science, Osaka University Graduate School of Medicine.

\section{Results}

Table 3 shows the baseline characteristics of subjects according to the number of risk factors in 1995. The mean age of all subjects was 40.6 years. The proportion of subjects without risk factors was $51.6 \%$, and the proportions of subjects with one or more risk factor, two or more risk factors, three or more risk factors, and four risk factors were $48.4,17.5,3.8$, and $0.2 \%$, respectively. In the singlerisk-factor group, dyslipidemia had the highest prevalence among the four risk factors. In the 2-risk-factor group, the prevalence of the combination of dyslipidemia and obesity was about $50 \%$, and in the 3-risk-factor group, the prevalence of the combination of dyslipidemia and obesity and hypertension was about $80 \%$.

Among 6477 subjects (excluding subjects in the 0-riskfactor group), 1907 subjects had recovered from at least one risk factor 9 years later. Table 4 shows the ORs for the recovery from risk factors according to the baseline HPI and eight health practices. The result for the 4-risk-factor group is not shown because there were so few subjects and the reliability of the analysis was low. In the single- and 2risk-factor groups, the ORs of recovery increased as the HPI increased ( $P$ for trend 0.01 and 0.02 , respectively). In the single-risk-factor group, the OR of recovery was higher in the good health practice group than in the poor health practice group (OR 1.27; 95\% CI 1.03, 1.57). In the 2- and $\geq 2$-risk-factor groups, the ORs of recovery were higher in the moderate health practice group (OR 1.29; 95\% CI 1.04, 1.60 and OR $1.21 ; 95 \%$ CI $1.00,1.45$, respectively). In Model 2, we found variables with a significant relation to the recovery in some groups. In the single-risk-factor group, those who ate nutritionally balanced diets or those who maintained moderate levels of mental stress had higher ORs of recovery than each of those groups with poor health practices (OR 1.24; 95\% CI 1.07, 1.45 and OR 1.19 ; $95 \%$ CI $1.03,1.38$, respectively). In the $2-$ and $\geq 2$ risk-factor groups, non-smokers (OR 1.26; 95\% CI 1.03, 1.53 , and OR $1.27 ; 95 \%$ CI $1.07,1.51$, respectively) or those who worked fewer than $9 \mathrm{~h}$ per day (OR 1.25; 95\% CI 1.02, 1.54 and OR $1.25 ; 95 \%$ CI 1.05, 1.49, respectively) had higher ORs of recovery. In the 3 - and $\geq 3$-riskfactor groups, we found no variables with a significant relation to the recovery.

\section{Discussion}

This study was initiated with the aim of clarifying how lifestyle at baseline influences the recovery from risk factors after 9 years. The results show that the relation between the recovery from risk factors and overall lifestyle differs according to the initial number of risk factors. In the 
Table 3 Baseline characteristics of the 13,394 male workers according to the number of risk factors (Japan, 1995)

\begin{tabular}{|c|c|c|c|c|c|c|}
\hline \multirow[t]{2}{*}{ Baseline characteristics } & \multirow[t]{2}{*}{ Total } & \multicolumn{5}{|c|}{ Number of risk factors in 1995} \\
\hline & & 0 & 1 & 2 & 3 & 4 \\
\hline Number of subjects & 13,394 & 6,917 & 4,129 & 1,843 & 479 & 26 \\
\hline Age $(\text { years })^{\mathrm{a}}$ & $40.6(7.9)$ & $39.6(8.3)$ & $41.5(7.4)$ & 41.7 (7.1) & $43.0(6.8)$ & $45.4(6.9)$ \\
\hline \multicolumn{7}{|l|}{ Ten-year categories $^{\mathrm{b}}$} \\
\hline $20-29$ & $1,417(10.6)$ & $970(14.0)$ & $316(7.7)$ & $110(6.0)$ & $20(4.2)$ & $1(3.8)$ \\
\hline $30-39$ & $4,028(30.1)$ & $2,195(30.1)$ & $1,174(31.7)$ & $541(28.4)$ & $115(24.0)$ & $3(11.5)$ \\
\hline $40-49$ & $6,512(48.6)$ & 3,079 (48.6) & $2,156(44.5)$ & $991(52.2)$ & $272(56.8)$ & $14(53.8)$ \\
\hline $50-59$ & $1,437(10.7)$ & $673(10.7)$ & $483(9.7)$ & $201(11.7)$ & $72(15.0)$ & $8(30.8)$ \\
\hline \multicolumn{7}{|l|}{ Risk factors ${ }^{\mathrm{b}}$} \\
\hline Obesity & $2,829(21.1)$ & $0(0.0)$ & $1,000(24.2)$ & $1,348(73.1)$ & $455(95.0)$ & $26(100.0)$ \\
\hline Hypertension & $1,965(14.7)$ & $0(0.0)$ & $781(18.9)$ & $737(40.0)$ & $421(87.9)$ & $26(100.0)$ \\
\hline Dyslipidemia & $4,208(31.4)$ & $0(0.0)$ & $2,255(54.6)$ & $1,462(79.3)$ & $465(97.1)$ & $26(100.0)$ \\
\hline Hyperglycemia & $354(2.6)$ & $0(0.0)$ & $93(2.3)$ & $139(7.5)$ & $96(20.0)$ & $26(100.0)$ \\
\hline \multicolumn{7}{|l|}{ Health practices ${ }^{b}$} \\
\hline Not smoking & $6,025(45.0)$ & $3,251(47.0)$ & $1,747(42.3)$ & $775(42.1)$ & $238(49.7)$ & $14(53.8)$ \\
\hline Not drinking more than $46 \mathrm{~g}$ ethanol per day & $12,735(95.1)$ & $6,666(96.4)$ & $3,877(93.9)$ & $1,715(93.1)$ & $453(94.6)$ & $24(92.3)$ \\
\hline Eating breakfast every morning & $10,209(76.2)$ & $5,299(76.6)$ & $3,159(76.5)$ & $1,370(74.3)$ & $366(76.4)$ & $15(57.7)$ \\
\hline Sleeping $7-8 \mathrm{~h}$ per night & $6,356(47.5)$ & $3,244(46.9)$ & $2,033(49.2)$ & $845(45.8)$ & $219(45.7)$ & $15(57.7)$ \\
\hline Working $9 \mathrm{~h}$ or less per day & $5,097(38.1)$ & $2,659(38.4)$ & $1,562(37.8)$ & $689(37.4)$ & $175(36.5)$ & $12(46.2)$ \\
\hline Exercising at least twice a week & $2,126(15.9)$ & $1,154(16.7)$ & $637(15.4)$ & $261(14.2)$ & $70(14.6)$ & $4(15.4)$ \\
\hline Eating a nutritionally balanced diet & $4,883(36.5)$ & $2,527(36.5)$ & $1,536(37.2)$ & $650(35.3)$ & $159(33.2)$ & $11(42.3)$ \\
\hline Keeping a moderate level of mental stress & $6,629(49.5)$ & $3,486(50.4)$ & $2,043(49.5)$ & $877(47.6)$ & $213(44.5)$ & $10(38.5)$ \\
\hline Health Practice Index (HPI) ${ }^{a}$ & $4.05(1.52)$ & $4.09(1.52)$ & $4.04(1.5)$ & $3.93(1.53)$ & $4.05(1.43)$ & $4.11(1.64)$ \\
\hline \multicolumn{7}{|l|}{ HPI category ${ }^{b}$} \\
\hline Good (6-8) & $2,324(17.4)$ & $1,257(18.2)$ & $702(17.0)$ & $288(15.6)$ & $72(15.0)$ & $5(19.2)$ \\
\hline Moderate (4-5) & $5,923(44.2)$ & $3,117(45.1)$ & $1,815(44.0)$ & $774(42.0)$ & $207(43.2)$ & $10(38.5)$ \\
\hline Poor $(0-3)$ & $5,147(38.4)$ & $2,543(36.8)$ & $1,612(39.0)$ & $781(42.4)$ & $200(41.8)$ & $11(42.3)$ \\
\hline
\end{tabular}

${ }^{a}$ Data are given as the mean with the standard deviation in parenthesis

b Data are given as frequency with the percentage in parenthesis

single-risk-factor group, the good health practice group had a significantly higher OR of recovery than the poor health practice group, and there was a significant linear trend, indicating that a good overall lifestyle is effective in terms of recovery from a single risk factor. In the 2 - and $\geq 2$-riskfactor groups, the moderate health practice group had significantly higher ORs of recovery than the poor health practice group. However, a significant linear trend was obtained only in the 2-risk-factor group. These results suggest that the effect of a good overall lifestyle on the recovery decreases for subjects with severe multiple risk factors, although a good overall lifestyle is effective for the recovery for subjects with two risk factors.

In almost all multiple-risk-factor groups, non-smoking was positively associated with recovery from risk factors. Non-smokers often show about a 1.2-fold higher recovery than smokers. Some studies have reported that non-smokers have a lower incidence of metabolic syndrome than those with other smoking statuses $[13-15,17,20]$. The results of our study agree with these findings, suggesting that non-smoking is important in the recovery from multiple risk factors.

Working $9 \mathrm{~h}$ or less per day was positively associated with recovery from risk factors in the 2 - and $\geq 2$-risk-factor groups. However, associations were not obtained for subjects with severe multiple risk factors, suggesting that working $\leq 9 \mathrm{~h}$ per day is important for subjects with two risk factors, although the effect of working $\leq 9 \mathrm{~h}$ per day decreases for subjects with severe multiple risk factors.

In the single-risk-factor group, the incidence of recovery in subjects with good health practices who eat a nutritionally balanced diet or maintain a moderate level mental stress was higher than that in subjects with poor health practices. However, these lifestyles were not associated with recovery from risk factors in the multiple-risk-factor groups. This result suggests that, although it is possible for subjects with a single risk factor to recover from risk factors by maintaining good eating habits and moderate 


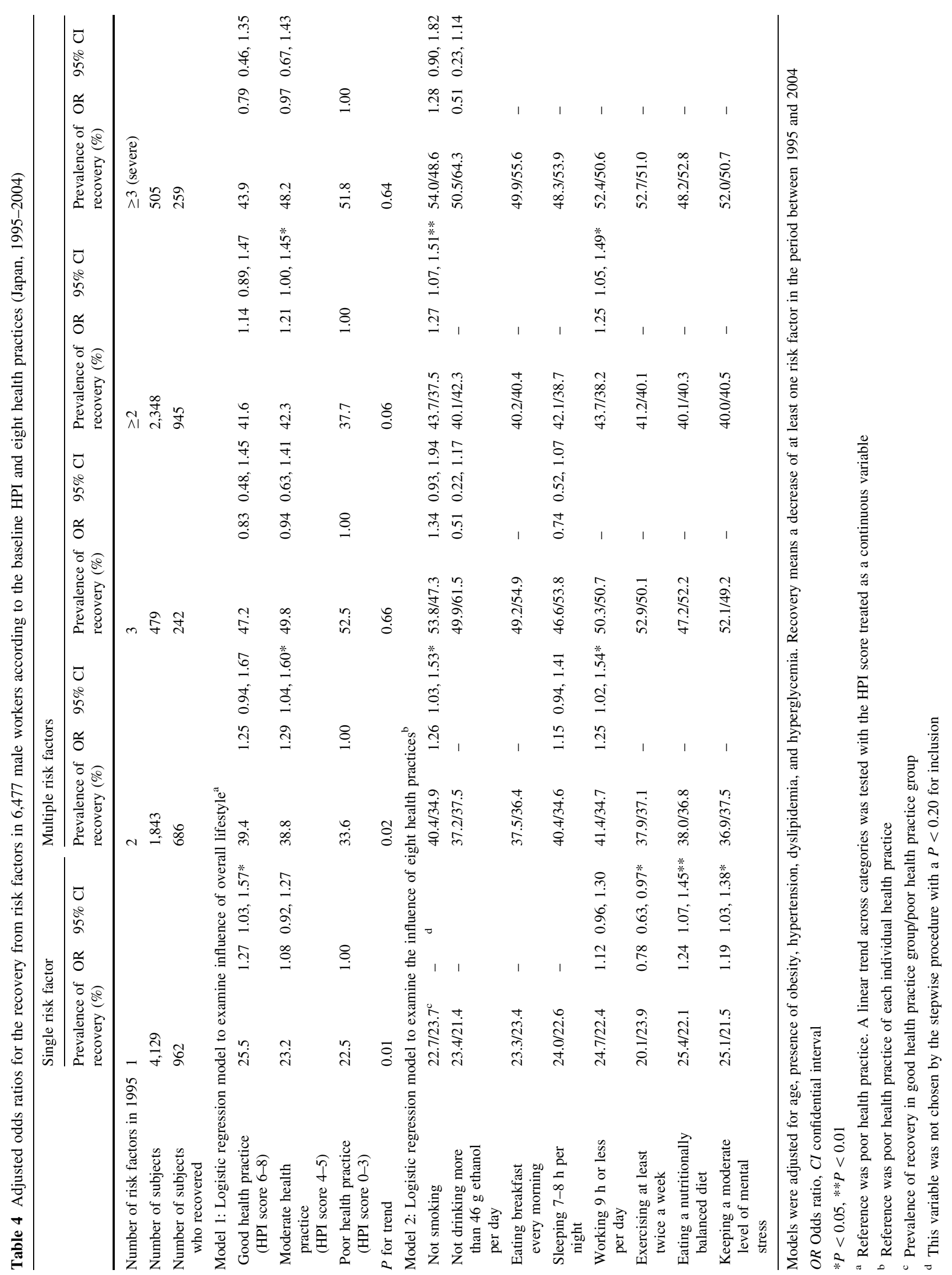


mental stress levels, it is difficult for subjects with multiple risk factors to recover from risk factors solely by maintaining these health practices.

Exercising at least twice a week was negatively associated with recovery from the risk factor in the single-riskfactor group. There were also no associations with the recovery in multiple-risk-factor groups. Generally, regular physical exercise is recommended for the prevention of metabolic diseases and metabolic syndrome, and positive effects of physical exercise on metabolic syndrome have been reported in previous studies [13,15-18]. We believe that discontinuous exercise habits can explain our results. The number of subjects who changed their health practices was not small because the follow-up period was long in this study. We examined the continuousness of the lifestyle by using longitudinal cohort data, which form the basis of the data used in this study, and found that exercise habits are not continued easily compared with the other seven health practices. For example, the continuance rate of good health practice over the 9 years was $13.7 \%$ for exercise habits compared to 30.2 and $19.5 \%$ for eating a nutritionally balanced diet and working time, respectively. To clarify the effect of exercise habits, it would be necessary to consider changes in lifestyle over the 9 years.

We found that the relation between the recovery from risk factors and lifestyle differed depending on the initial number of risk factors each subject had. For subjects who initially had a single risk factor, a good overall lifestyle was found to promote the recovery from the risk factors. In this context, maintaining good dietary habits and managing stress were found to be especially effective in the recovery. For subjects who initially had multiple risk factors, a good overall lifestyle was found to promote the recovery, with non-smoking and limiting working hours especially effective. However, the relation between recovery from multiple risk factors and maintaining good dietary habits and managing stress was found to be less in these subjects than in those with a single risk factor. This result suggests that the effects of dietary modification and mental stress reduction were less in subjects who initially had multiple risk factors than in those with a single risk factor. For subjects with severe multiple risk factors, no significant relations were obtained, possibly due to methodological issues. Only the age and risk factors were adjusted in this study; consequently, other factors, such as family history, may have influenced the results. The number of subjects who had treatments for risk factors cannot be considered to be too small as the follow-up period was quite long. A consideration of data during the follow-up period and other various factors would be necessary to examine the long-term influences of lifestyle on severe multiple risk factors. However, our results do show that the mechanism of a group of multiple risk factors is different from the mechanism of a single risk factor, and they have clarified one of the methods for recovery from the group of the multiple risk factors.

The large study sample and the long-term follow-up are the strengths of our study; however, there are several limitations. Firstly, much of the data were excluded due to missing values, subjects dropping out of the follow-up due to resignation or job changes, subjects not going for their medical checkup, among others. Accordingly, subjects who underwent checkups in 2004 represented 26\% of all subjects who underwent medical checkups in 1995. Compared with non-participants who had missing values or dropped out, there was a trend for participants to be in the 30- to 40-year age group and to have a better lifestyle. We did not adjust for any bias of lifestyle although age was adjusted. A non-participant's ratio of poor lifestyle was higher than that of a participant. As such a nonparticipant has a higher chance of dropping out due to deterioration in metabolic syndrome caused by poor lifestyle. It is believed that the recovery rate in the poor lifestyle group would be lower if it could be followed for 9 years and included non-participants. Therefore, in this study there is a possibility that the estimated difference of the effect between a good lifestyle and poor lifestyle is smaller than the actual difference.

Only data from 1995 and 2004 were examined. Therefore, changes in lifestyle during this period were not considered. In addition, the causal relation between a change in health status and changes in lifestyle was not clarified. Although lifestyle intervention was not given at the checkup, changes in lifestyle during this period could have occurred. Such changing patterns in health status and lifestyles should be examined, and this causal relation should be clarified.

A number of definitions of metabolic syndrome have been reported in studies worldwide [21, 22]. In Japan, the National Metabolic Syndrome Criteria study Group has proposed new criteria for metabolic syndrome in Japan [23]. However, the criteria are still being discussed because evidence in the Japanese population is at yet insufficient. Therefore, we used the definitions of abnormality in the criteria of each Japan Society as definitions of risk factors of metabolic syndrome. The National Cholesterol Education Program Adult Treatment Panel III [21] and the Japanese criteria emphasize the presence of visceral obesity and include measurements of waist circumference (WC) instead of BMI. Visceral obesity has been found to have a higher correlation with cardiovascular risk factors, such an insulin resistance, hyperinsulinemia, glucose intolerance, type-2 diabetes, dyslipidemia, and hypertension, when compared to obesity defined by BMI [24-26]. In this study, BMI was used instead of WC because WC data were not collected. In Japanese checkups, WC measurements are not regularly carried out. Therefore, existing data cannot be 
easily used to estimate metabolic syndrome prevalence by criteria including visceral obesity.

Recent studies have reported an association between genetic type and metabolic syndrome [3, 27-29]. The effect of lifestyle on hypertension according to genetic type has been previously clarified [30]. Future studies should clarify the effect of lifestyle on the recovery from metabolic syndrome taking genetic type into consideration.

For the prevention of metabolic syndrome, it is important to clarify factors that influence not only the recovery but also deterioration. We are now examining factors that influence deterioration and plan to report our findings in the future.

In conclusion, we have shown that there is relation between the recovery from a group of multiple risk factors and lifestyle. This relation is different from that for the recovery from a single risk factor, and it differs depending on the number of risk factors. Our results provide evidence that effective modifications in lifestyles for people with a group of multiple risk factors are different from effective modifications for people with a single risk factor.

\section{References}

1. Gami AS, Witt BJ, Howard DE, Erwin PJ, Gami LA, Somers VK, et al. Metabolic syndrome and risk of incident cardiovascular events and death: a systematic review and meta-analysis of longitudinal studies. J Am Coll Cardiol. 2007;49:403-14.

2. Takeuchi H, Saitoh S, Takagi S, Ohnishi H, Ohhata J, Isobe T, et al. Metabolic syndrome and cardiac disease in Japanese men: applicability of the concept of metabolic syndrome defined by the National Cholesterol Education Program-Adult Treatment Panel III to Japanese men-the Tanno and Sobetsu Study. Hypertens Res. 2005;28:203-8.

3. Yamada Y, Kato K, Hibino T, Yokoi K, Matsuo H, Segawa T, et al. Prediction of genetic for metabolic syndrome. Atherosclerosis. 2007;191:298-304.

4. Iso H, Date C, Wakai K, Fukui M, Tamakoshi A, Fukui M, et al. The relationship between green tea and total caffeine intake and risk for self-reported type 2 diabetes among Japanese adults. Ann Intern Med. 2006;144:554-62.

5. Nakanishi N, Okamoto M, Nakamura K, Suzuki K, Tatara K. Cigarette smoking and risk for hearing impairment: a longitudinal study in Japanese male office workers. J Occup Environ Med. 2000;42:1045-9.

6. Waki K, Noda M, Sasaki S, Matsumura Y, Takahashi Y, Isogawa A, et al. Alcohol consumption and other risk factors for selfreported diabetes among middle-aged Japanese: a populationbased prospective study in the JPHC study cohort I. Diabet Med. 2005;22:323-31.

7. Morimoto K, Takeshita T, Take-uchi T, Maruyama S, Ezoe S, Mure K, et al. Chromosome alterations in peripheral lymphocytes as indices of lifestyle and genotoxicity. Int Arch Occup Environ Health. 1993;65[Suppl 1]:S37-41.

8. Kusaka Y, Kondou H, Morimoto K. Healthy lifestyles are associated with higher natural killer cell activity. Prev Med. 1992;21:602-15.
9. Morimoto K, Takeshita T, Inoue-Sakurai C, Maruyama S. Lifestyles and mental health status are associated with natural killer cell and lymphokine-activated killer cell activities. Sci Total Environ. 2001;270:3-11.

10. Morimoto K, Takeshita T, Nanno M, Tokudome S, Nakayama K. Modulation of natural killer cell activity by supplementation of fermented milk containing Lactobacillus casei in habitual smokers. Prev Med. 2005;40:589-94.

11. Mure K, Takeshita T, Takeuchi T, Morimoto K. Urinary mutagens and lifestyle factors. Prev Med. 1996;25:569-74.

12. Shirakawa T, Morimoto K. Lifestyle effect on total IgE. Lifestyles have a cumulative impact on controlling total IgE levels. Allergy. 1991;46:561-9.

13. Wannamethee SG, Shaper AG, Whincup PH. Modifiable lifestyle factors and the metabolic syndrome in older men: effects of lifestyle changes. J Am Geriatr Soc. 2006;54:1909-14.

14. Holme I, Tonstad S, Sogaard AJ, Larsen PGL, Haheim LL. Leisure time physical activity in middle age predicts the metabolic syndrome in old age: results of a 28-year follow-up of men in the Oslo study. BMC Public Health. 2007;7:154. doi: 10.1186/1471-2458-7-154.

15. Park HS, Oh SW, Cho SI, Choi WH, Kim YS. The metabolic syndrome and associated lifestyle factors among South Korean adults. Int J Epidemiol. 2004;33:328-36.

16. Villegas R, Creagh D, Hinchion R, O'Halloran D, Perry IJ. Prevalence and lifestyle determinants of the metabolic syndrome. Ir Med J. 2004;97:300-3.

17. Zhu S, St-Onge MP, Heshka S, Heymsfield SB. Lifestyle behaviors associated with lower risk of having the metabolic syndrome. Metabolism. 2004;53:1503-11.

18. Orchard TJ, Temprosa M, Goldberg R, Haffner S, Ratner R, Marcovina $S$, et al. The effect of metformin and intensive lifestyle intervention on the metabolic syndrome: the Diabetes Prevention Program randomized trial. Ann Intern Med. 2005;142:611-9.

19. Morimoto K. Lifestyle and health (in Japanese). Jpn J Hyg. 2000;54:572-91.

20. Ishizaka N, Ishizaka $\mathrm{Y}$, Toda $\mathrm{E}$, Hashimoto H, Nagai R, Yamakado M. Association between cigarette smoking, metabolic syndrome, and carotid arteriosclerosis in Japanese individuals. Atherosclerosis. 2005;181:381-8.

21. Expert Panel on Detection, Evaluation, and Treatment of High Blood Cholesterol in Adults (2001) Executive Summary of the Third Report of the National Cholesterol Education Program (NCEP) Expert Panel on Detection, Evaluation, and Treatment of High Blood Cholesterol in Adults (Adult Treatment Panel III). JAMA 285:2486-97.

22. Alberti KG, Zimmet PZ. Definition, diagnosis and classification of diabetes mellitus and its complications. Part 1: diagnosis and classification of diabetes mellitus provisional report of a WHO consultation. Diabet Med. 1998;15:539-53.

23. Matsuzawa Y. Metabolic syndrome: definition and diagnostic criteria in Japan (in Japanese). J Jpn Soc Int Med. 2005;94:188203.

24. Sonmez K, Akcakoyun M, Akcay A, Demir D, Duran NE, Gençbay M, et al. Which method should be used to determine the obesity, in patients with coronary artery disease? (body mass index, waist circumference or waist-hip ratio). Int $\mathrm{J}$ Obes. 2003;27:341-6.

25. Wang Y, Rimm EB, Stampfer MJ, Willett WC, Hu FB. Comparison of abdominal adiposity and overall obesity in predicting risk of type 2 diabetes among men. Am J Clin Nutr. 2005;81:55563.

26. Dalton M, Cameron AJ, Zimmet PZ, Shaw JE, Jolley D, Dunstan DW, et al. Waist circumference, waist-hip ratio and body mass 
index and their correlation with cardiovascular disease risk factors in Australian adults. J Intern Med. 2003;254:555-63.

27. Tanigawa $T$, Iso $H$, Yamagishi K, Muraki I, Kawamura N, Nakata A, et al. Association of lymphocyte sub-populations with clustered features of metabolic syndrome in middle-aged Japanese men. Atherosclerosis. 2004;173:295-300.

28. Gable DR, Hurel SJ, Humphries SE. Adiponectin and its gene variants as risk factors for insulin resistance, the metabolic syndrome and cardiovascular disease. Atherosclerosis. 2006;188: 231-44.
29. Semple RK, Chatterjee VK, O'Rahilly S. PPAR gamma and human metabolic disease. J Clin Invest. 2006;116:581-9.

30. Itoh T, Matsumoto M, Nakamura M, Okada A, Shirahashi N, Hougaku $\mathrm{H}$, et al. Effects of daily alcohol intake on the blood pressure differ depending on an individual's sensitivity to alcohol: oriental flushing as a sign to stop drinking for health reasons. J Hypertens. 1997; 15:1211-7. 